Case report

\title{
Valve-sparing aortic root replacement and aortic valve repair in a patient with acromegaly and aortic root dilatation
}

\author{
Karel Van Praet, Jeffrey I. Mechanick, Vicente Orozco, Allan S. Stewart \\ Mount Sinai Hospital, New York City, USA
}

Received 2 February 2015, Revised 31 March 2015, Accepted 18 April 2015

(C) 2015, Van Praet K., Mechanick J.I., Orozco V., Stewart A.S.

(C) 2015, Russian Open Medical Journal

Abstract: Aortic regurgitation and dilatation of the aortic root and ascending aorta are severe complications of acromegaly. The current trend for management of an aortic root aneurysm is valve-sparing root replacement as well as restoring the diameter of the aortic sinotubular junction (STJ) and annulus. Our case report supports the recommendation that in patients with acromegaly, severe aortic root involvement may indicate the need for surgery.

Keywords: aorta, aortic operation, aortic root, aortic valve repair, cardiac surgery, endocrinology, acromegaly, pituitary tumor

Cite as Van Praet K, Mechanick JI, Orozco V, Stewart AS. Valve-sparing aortic root replacement and aortic valve repair in a patient with acromegaly and aortic root dilatation. Russian Open Medical Journal 2015; 4: e0303.

Correspondence to Dr. Allan S. Stewart. Address: 1190, Fifth Avenue, GP2W, NewYork, NY10029, USA. E-mail: karel.vanpraet@gmail.be. Phone: 212-659-6807. Fax: 212-659-6818

\section{Case Report}

A 51-year-old man with a history of hypertension, sickle cell trait, scoliosis, and pectus carinatum was referred to our department for surgical evaluation. For the past four years, the patient reported worsening shortness of breath, angina while exercising, fatigue, and chest pain. Physical examination revealed signs and symptoms of heart failure. On chest $x$-ray, the enlarged ascending aorta produced a convex contour of the right superior mediastinum and in the lateral view, there was loss of the retrosternal air space. An echocardiographic examination revealed a dilated aortic root $(4.1 \mathrm{~cm})$, mitral valve prolapse (grade II), mild mitral valve regurgitation (grade 2/4), mild aortic regurgitation, and left ventricular ejection fraction (LVEF) of $60 \%$. Medical therapy included an angiotensin-converting enzyme inhibitor and a calcium channel blocker. A CT chest confirmed aortic root dilatation measuring up to $4.2 \mathrm{~cm}$ and dilatation of the ascending thoracic aorta of $3.7 \mathrm{~cm}$ (Figure 1). He was acromegalic, with prognathism, glossomegaly and tall stature (Figure 2). Fasting growth hormone $(\mathrm{GH})$ and IgF-1 levels were increased to 115 and 338 units, respectively. Ahead CT showed an aggressive pituitary lesion with extrasellar extension consistent with a $\mathrm{GH}$-secreting tumor. The patient underwent valve-sparing aortic root replacement with a Vascutek ${ }^{\mathrm{R}}$ Valsalva $^{\mathrm{TM}}$ graft and aortic valve repair using aortic arch and right atrial cannulation and antegrade del Nido cardioplegia for heart protection. We performed a limited median sternotomy. The aorta was transected at the sinotubular junction (STJ) and the root was prepared in the standard fashion. For optimal exposure, the aorta was completely transsected at the STJ 5 to $10 \mathrm{~mm}$ above the commissures and stay sutures were placed in order to facilitate mobilisation of the root. We applied a pragmatic approach in choosing graft size and took a graft approximately 4 to $5 \mathrm{~mm}$ larger than the diameter of the left ventricular outflow tract (LVOT). The LVOT was sized and found to be $25 \mathrm{~mm}$. Subsequently, a $30 \mathrm{~mm}$ Valsalva ${ }^{\text {TM }}$ graft was selected for reconstruction. The graft was then sutured to the aortic root, carefully following the insertion line of the aortic cusps. We started in the center of the sinus and worked our way up to the commissures. By doing so, it was easy to accommodate the exact height of the commissure in the graft. This reduction of sinus dimensions led to some degree of aortic cusp prolapse. An effective cusp height of $8 \mathrm{~mm}$ corresponded to a similar height on preoperative echocardiograms and resulted in a normal configuration of the aortic cusps. In order to achieve this height we shortened the free margin of the aortic cusp by placing plication sutures using $6 / 0$ prolene. Reimplantation of the coronaries was done in a standard fashion. We kept the openings in the graft relatively small, that is approximately the size of the coronary ostia. Finally, the aortic graft was trimmed to adequate length and anastomosed to the ascending aorta (Figure 3). Finally, the patient was easily weaned from bypass and postoperative transoesophageal echocardiography (TEE) confirmed perfect coaptation of the valve leaflets with absence of aortic regurgitation. Extracorporeal circulation time, cross clamp time and reperfusion time were 101 minutes, 41 minutes and 35 minutes respectively. A 6 days postoperative contrast enhanced CT chest at the level of the STJ of the aortic root reveals a maximal aortic diameter of $31.45 \mathrm{~mm}$ (Figure 4). The patient was discharged home on day 7 on angiotensin-converting enzyme inhibitor alone and experienced an uneventful recovery. A 4 months postoperative echocardiographic examinations howed a persistently reduced aortic diameter of $3.1 \mathrm{~cm}$. At 6 months follow-up our patient was still free of complaints and reported a significantly improved quality of life. 


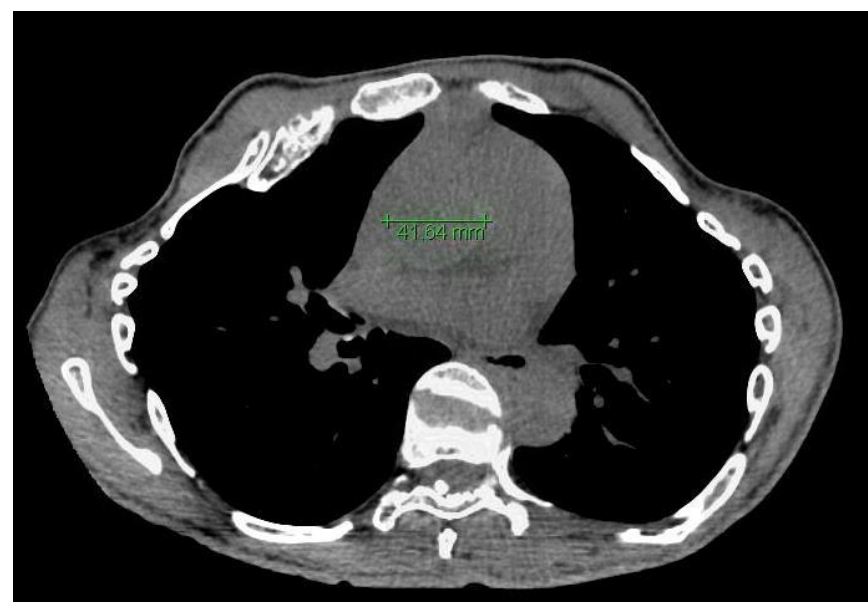

Figure 1. Preoperative CT chest with out contrast at the level of STJ of the aortic root revealing a maximal aortic diameter of $41.64 \mathrm{~mm}$

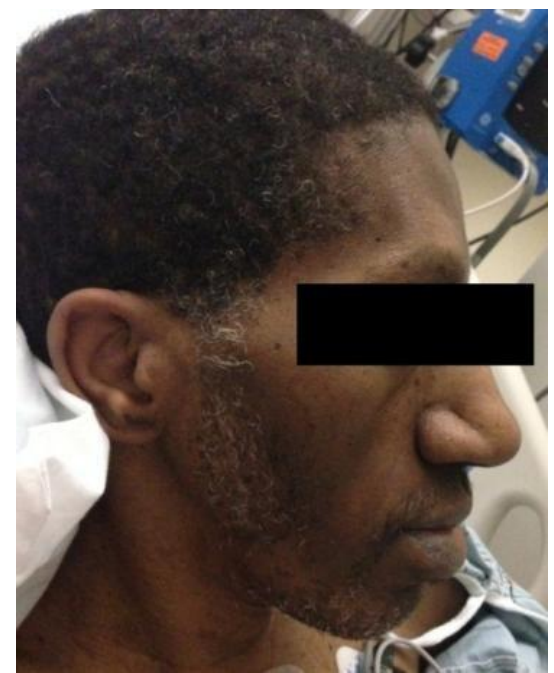

Figure 2. Our patient with acromegaly showing prognathism and glossomegaly

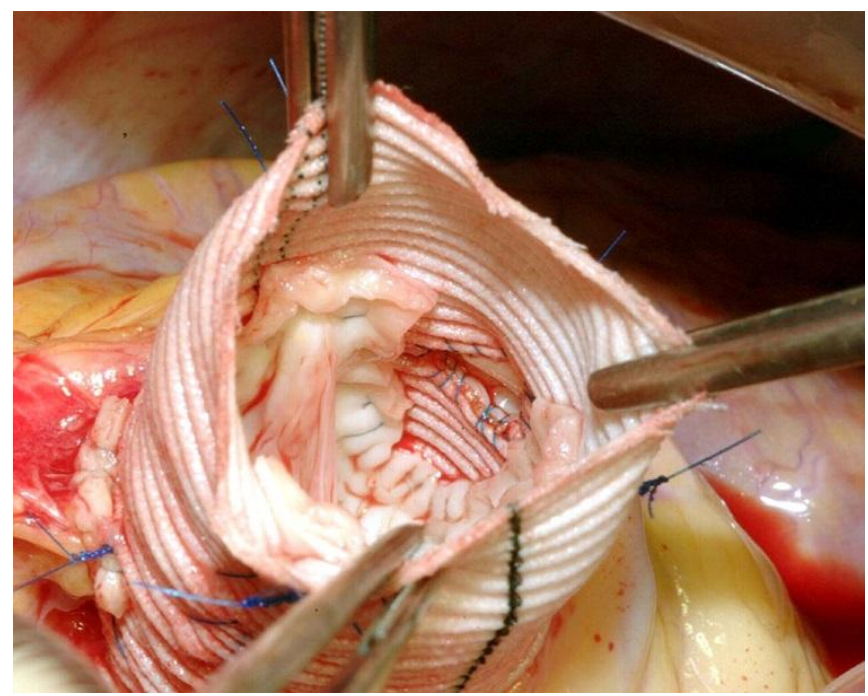

Figure 3. Intraoperative image of the Valsalva ${ }^{\mathrm{TM}}$ graft and aortic valve repair

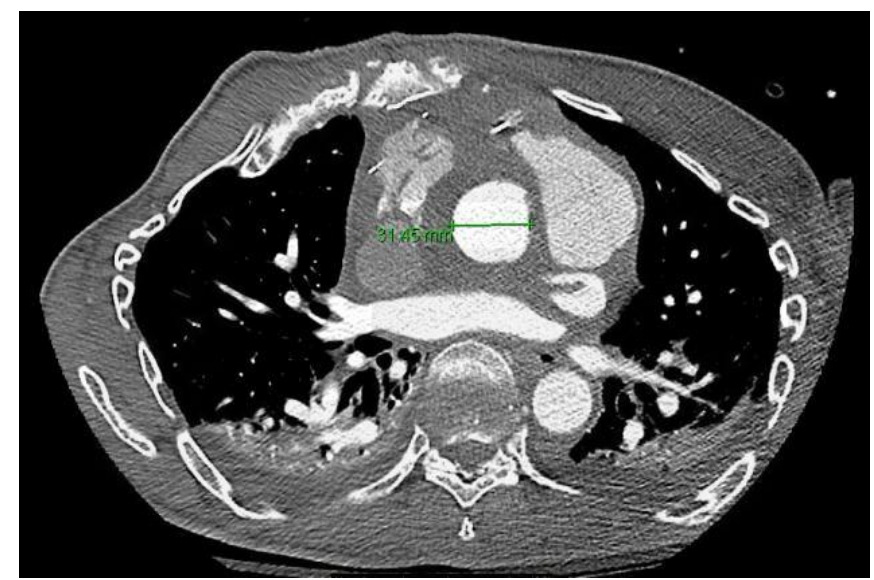

Figure 4. 6 days postoperative contrast enhanced CT chest at the level of the STJ of the aortic root revealing a maximal aortic diameter of $31.45 \mathrm{~mm}$

\section{Discussion}

Acromegaly is an uncommon disease with alow incidence. Our case presented with a dilated aortic root, an ascending aortic aneurysm, and severe aortic regurgitation that required valvesparing aortic root replacement and aortic valve repair. Prolonged exposure to $\mathrm{GH}$ affects the aortic root, the aortic valve, and the myocardium. Vander Klaauw et al. [1] showed that aortic root dimensions at the STJ junction and ascending aorta increase in patients with acromegaly, compared with healthy controls, after patients with aortic insufficiency were excluded from their study. It is generally regarded that abnormal production of extracellular matrix, coupled with myxoid degeneration are responsible for cardiac complications in acromegaly. Thisisdue to the effects of $\mathrm{GH}$ on alterations in collagen deposition in the aorta. Histologic findings in the report of Maffei et al. [2] include myofibrillar derangement with myxomatous degeneration, depletion in the extracellular matrix of the internal and external lamina, and mucopolysaccharide accumulation in the aortic wall. Izgi et al. [3] recommends that patients with acromegaly should be screened for aortic pathologies by evaluating their aortic root and ascending aorta dimensions. Our case report supports this recommendation and further, that severe aortic root involvement may indicate the need for surgery. The current trend for management of aortic root aneurysm is valve-sparing root replacement as well as restoring the diameter of the aortic STJ and annulus, but this is still controversial. Native aortic valve preservation results in maintenance of proper hemodynamics and prevention of thromboembolic complications by avoiding the use of aprosthetic valve and anticoagulation [4]. When attempting to preserve aortic valve function crucial factors includead equatesize and morphology of the leaflets, diameter of the aortic annulus, and diameter of the STJ [5]. The native valve can be spared if aortic regurgitation is secondary to dilatation of the aortic root or the STJ if the aortic valve leaflets are grossly normal [6]. Badiu et al. [7] showed that this procedure can be performed with an acceptable morbidity, mortality, and long-term survival positioning valvesparing aortic root replacement as the first choice intervention in patients undergoing aortic root replacement. 


\section{Conclusion}

In conclusion, dilatation of the aortic root and ascending aorta and aortic regurgitation are frequent complications of acromegaly. We suggest that in patients with acromegaly, careful radiographic evaluation of the aortic root and ascending aorta should be performed. When surgical repair of the aortic valve and root is indicated, valve-sparing aortic root replacements should then be the first choice of intervention.

\section{Conflict of interest: none declared.}

\section{References}

1. Van der Klaauw AA, Bax JJ, Smit JW, Holman ER, Delgado V, Bleeker $\mathrm{GB}$, et al. Increased aortic root diameters in patients with acromegaly. Eur J Endocrinol 2008; 159(2): 97-103. (doi: 10.1530/EJE-08-0138) (PMID: 18495693)

2. Maffei P, Menegazzo C, Michelotto M, Salmaso R, Martini C, De Carlo $\mathrm{E}$, et al. Sudden death due to aortic rupture in acromegaly. Heart Vessels 2008; 23(1): 71-74. (doi: 10.1007/s00380-007-1012-y) (PMID: 18273550)

3. Izgi C, CevikC, Nugent K. Annuloaortic ectasia and ascending aortic aneurysm as a cardiovascula manifestation of acromegaly. Cardiovasc Intervent Radiol 2010; 33: 438-440. (doi: 10.1007/s00270-009-9631-6) (PMID: 19548029)

4. David TE, Feindel CM, Webb GD, Colman JM, Armstrong S, Maganti M. Aortic valve preservation in patients with aortic root aneurysm: results of the reimplantation technique. Ann Thorac Surg 2007; 83(2): S732S735. (PMID: 17257917) (doi: 10.1016/j.athoracsur.2006.10.080)

5. Neragi-Miandoab S. Repair of dilated aortic root and sinotubular junction using astabilizer ring. Recent Pat Cardiovasc Drug Discov 2012; 7(2): 134-140. (doi: 10.2174/157489012801227229) (PMID: 22494027)

6. Morimoto N, Matsumori M, Tanaka A, MunakataH, Okada K, Okita Y. Adjustment of sinotubular junction for aortic insufficiency secondary to ascending aortic aneurysm. Ann Thorac Surg 2009; 88(4): 12381243. (doi: 10.1016/j.athoracsur.2009.05.048) (PMID: 19766813)

7. Badiu CC, Deutsch MA, Sideris C, Krane M, Hettich I, Voss B, et al. Aortic root replacement: comparison of clinical outcome between different surgical techniques. Eur J Cardiothorac Surg 2014; 46(4): 685692. (doi: 10.1093/ejcts/ezt647) (PMID: 24497393)

\section{Authors:}

Karel Van Praet - Surgery Intern from University Hospitals Leuven Belgium (KULeuven), Department of Cardiothoracic Surgery, Mount Sinai Hospital, New York City, USA.

Jeffrey I. Mechanick - Attending Physician, Department of Internal Medicine, Mount Sinai Hospital, New York City, USA.

Vicente Orozco - Chief Resident Cardiothoracic Surgery, Department of Cardiothoracic Surgery, Mount Sinai Hospital, New York City, USA.

Allan S. Stewart - Attending Physician (Attending Cardiothoracic Surgeon), Department of Cardiothoracic Surgery, Mount Sinai Hospital, New York City, USA. 